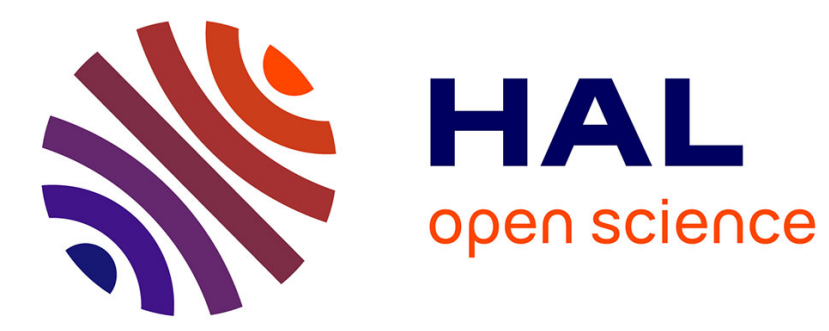

\title{
Polynomial growth, recurrence and ergodicity for random walks on locally compact groups and homogeneous spaces
}

\author{
Yves Guivarc'H, C. Robinson Edward Raja
}

\section{- To cite this version:}

Yves Guivarc'H, C. Robinson Edward Raja. Polynomial growth, recurrence and ergodicity for random walks on locally compact groups and homogeneous spaces. Workshop on Boundaries, Jun 2009, Graz, Austria. pp.65-74. hal-00462021v2

\section{HAL Id: hal-00462021 \\ https://hal.science/hal-00462021v2}

Submitted on 15 Mar 2010

HAL is a multi-disciplinary open access archive for the deposit and dissemination of scientific research documents, whether they are published or not. The documents may come from teaching and research institutions in France or abroad, or from public or private research centers.
L'archive ouverte pluridisciplinaire HAL, est destinée au dépôt et à la diffusion de documents scientifiques de niveau recherche, publiés ou non, émanant des établissements d'enseignement et de recherche français ou étrangers, des laboratoires publics ou privés. 


\title{
Polynomial growth, recurrence and ergodicity for random walks on locally compact groups and homogeneous spaces
}

\author{
Y. Guivarc'h and C.R.E. Raja
}

\begin{abstract}
Let $\mathrm{G}$ be a locally compact group, E a homogeneous space of G. We discuss the relations between recurrence of a random walk on $G$ or E, ergodicity of the corresponding transformations and polynomial growth of $\mathrm{G}$ or $\mathrm{E}$. We consider the special case of linear groups over local fields.
\end{abstract}

Keywords: Random walk ; ergodicity homogeneous spaces ; polynomial growth MSC(2000): 22E30, 31C12, 37A17, 37A50

\section{Introduction}

Let $G$ be a locally compact and separable group, $\lambda_{G}$ be a left Haar measure on $G$. We denote by $\mu$ a given probability measure on $G$, by $G_{\mu}$ the closed subgroup generated by its support. We study recurrence properties of random walks defined by $\mu$, either on $G$, or on homogeneous spaces of $G$. We also discuss ergodicity of associated homeomorphisms on path spaces. This paper can be considered as an introduction to the more complete article 17] where detailed proofs are given. For basic informations and results we refer to the surveys [7], [14] and to the books [6], [12], [29], [30]. We thank F. Ledrappier P. Bougerol, Y. Derriennic and T. Steger for useful comments on ergodicity of bilateral Markov shifts with infinite invariant measure.

\section{Polynomial growth}

Definition 1 We say that $G$ has polynomial growth of degree at most $d \geq 0$ if for any compact neighbourhood $W$ of $e$, there exists $C_{W}>0$ such that for every $n \in \mathbb{N}$

$$
\lambda_{G}\left(W^{n}\right) \leq C_{W} n^{d} .
$$

A typical example of polynomial growth is the following : $N$ is a nilpotent compactly generated group, $K$ is a compact group of automorphisms of $N$ and $G$ is a semi-direct product of $K$ and $N$. Then $d$ can be calculated in terms of the descending series of $N$ (See[10]). We give now a few structural facts.

It is well known that polynomial growth of $G$ implies amenability and unimodularity for $G$ as well as for its closed subgroups. A fundamental result of [9] says that if $G$ is finitely generated with polynomial growth then $G$ is virtually nilpotent. If $G$ is locally compact and compactly generated with polynomial growth then $G$ has a compact normal subgroup $K$ such that $G / K$ is a Lie group (connected or not) [21]. The connected Lie groups with 
polynomial growth are the groups of rigid type [10]. Rigid type for a connected Lie group $G$ means that for every $g \in G$, the automorphism Adg of the Lie algebra of $G$ has only eigenvalues of modulus one.

\section{Transience and recurrence}

\section{1) Definitions}

We consider the product space $\Omega=G^{\mathbb{N}}$ (resp $\widehat{\Omega}=G^{\mathbb{Z}}$ ) endowed with the shift $\theta$ and the product measure $\mathbb{P}=\mu^{\mathbb{N}}$ (resp $\widehat{\mathbb{P}}=\mu^{\mathbb{Z}}$ ) and we denote by $X_{k}(\omega)$ the coordinates of $\omega \in \Omega(\operatorname{resp} \omega \in \widehat{\Omega})$. If $E$ is a locally compact $G$ - space and $x \in E$, the random walk on $E$ of law $\mu$, starting from $x$, is the sequence of random variables $S_{n}(\omega) x(n \in \mathbb{N} \cup\{0\}$ or $\mathbb{Z})$ defined by :

$$
\begin{gathered}
S_{0}(\omega) x=x \\
S_{n}(\omega) x=X_{n}(\omega) \cdots X_{1}(\omega) x \quad(n>0) \\
S_{n}(\omega) x=X_{n+1}^{-1}(\omega) \cdots X_{0}^{-1}(\omega) x \quad(n<0)
\end{gathered}
$$

If $E=G$, the asymptotic behaviour of $S_{n}(\omega) x$ is essentially independent of $x \in G$. This is not the case in general if $E \neq G$ and we will discuss the situation in terms of a Radon measure $\lambda$ quasi invariant under the $\mu$-action on $E$. In particular if $\lambda$ is a $\mu$-invariant measure $(\mu * \lambda=\lambda)$ we will consider on $\Omega \times E$ the measure $\widetilde{\lambda}=\mathbb{P} \otimes \lambda$. The skew product $(\Omega \times E, \widetilde{\theta}, \mathbb{P} \otimes \lambda)$, with $\widetilde{\theta}(\omega, x)=\left(\theta \omega, X_{1}(\omega) x\right)$ will play a basic role since $\widetilde{\lambda}$ is $\widetilde{\theta}$-invariant. If $E=G$, we will take $\lambda=\lambda_{G}$. We will need also to consider the extended bilateral shift $\widehat{\theta}$ on $\widehat{\Omega} \times E: \widehat{\theta}(\omega, x)=\left(\theta \omega, X_{1}(\omega) x\right)$ which leads to consider the bilateral random walk $S_{n}(\omega)$ on $E(n \in \mathbb{Z})$. The Markov operator $P$ on $C_{b}(E)$, the space of continuous bounded functions on $E$, which is defined by

$$
P \varphi(x)=\int \varphi(g x) d \mu(g)
$$

allows to express various quantities of probabilistic interest. For example if $A \subset E$, the expected number of visits of the random walk to $A$ is

$$
\int \sum_{0}^{\infty} 1_{A}\left(S_{n}(\omega) x\right) d \mathbb{P}(\omega)=\sum_{0}^{\infty} P^{k} 1_{A}(x) .
$$

If $E=G$, we have

$$
\sum_{0}^{\infty} P^{k} 1_{A}(e)=\sum_{0}^{\infty} \mu^{k}(A)
$$

where $\mu^{k}$ is the $k-t h$ convolution power of $\mu$. We will now mainly restrict to the case $E=G$, and we will come back to the general case in the last section. 
Definition 2 We will say that $\mu$ or $S_{n}(\omega)$ is recurrent on $G$ if for every neighbourhood $W$ of e we have $\mathbb{P}-$ a.e $: S_{n}(\omega) \in W$ infinitely often. If $\mathbb{P}$ - a.e, $S_{n}(\omega)$ escapes to infinity we will say that $\mu$ or $S_{n}(\omega)$ is transient.

It is easy to see that $S_{n}(\omega)$ is either transient or recurrent. On can show, using Hopf maximal ergodic lemma, that a necessary and sufficient condition for transience of $\mu$ is the existence of $W$, a relatively compact neighbourhood of $e$, such that $\sum_{0}^{\infty} \mu^{n}(W)<\infty$.

We will assume $\mu$ adapted i.e $G_{\mu}=G$, and also we will exclude the case $G=\mathbb{Z}$ and $\mu=\delta_{a}(a \in \mathbb{Z})$. The following important concept will be used below

Definition 3 Let $f$ be a Borel function on $G$. We say that $f$ is left $\mu$-harmonic (resp left $\mu$-superharmonic) if $\int f(h g) d \mu(h)=f(g)$, for any $g \in G$

(resp $f \geq 0$ and $\int f(h g) d \mu(h) \leq f(g)$ for any $g \in G$ )

We observe that we could have considered $S_{n}^{\prime}(\omega)=X_{1}(\omega) \cdots X_{n}(\omega)$, instead of $S_{n}(\omega)$. But the laws of $S_{n}(\omega)$ and $S_{n}^{\prime}(\omega)$ are equal to $\mu^{n}$. Then the above implies that recurrence (resp transience) of $S_{n}(\omega)$ is equivalent to recurrence (resp transience) of $S_{n}^{\prime}(\omega)$. Hence we can speak of $\mu$ being recurrent (resp transient).

If $\mu$ is transient and $u \in C_{c}^{+}(G)$, the non negative function $\sum_{0}^{\infty} P^{k} u(x)=\sum_{0}^{\infty}\left(\mu^{k} * \delta_{x}\right)(u)$ is continuous and left-superharmonic.

Then we have the

Proposition 1 Assume $\mu$ is as above. Then we have the equivalence

a) $S_{n}(\omega)$ is recurrent

b) Any continuous left $\mu$-superharmonic function is constant

c) $\widehat{\theta}$ is ergodic with respect to $\widehat{\mathbb{P}} \otimes \lambda_{G}$.

A natural connection beween $\widetilde{\theta}$-invariant functions and $\mu$-harmonic functions modulo $\lambda_{G}$ is as follows.

If $F \in \mathbb{L}^{\infty}\left(\Omega \times G, \mathbb{P} \otimes \lambda_{G}\right)$ satisfies $F o \widetilde{\theta}=F$ then the function $f \in \mathbb{L}^{\infty}(G)$ defined by

$$
f(g)=\int F(\omega, g) d \mathbb{P}(\omega)
$$

satisfies $\int f(h g) d \mu(h)=f(g)$, i.e $f$ is $\mu$-harmonic mod $\lambda_{G}$. As is well known, this correspondance is bijective. Ergodicity of $\widetilde{\theta}$ amounts to constancy of bounded $\mu$-harmonic functions. Ergodicity of $\widehat{\theta}$ is a stronger property. If $G=\mathbb{Z}^{3}$ it is easy to construct non trivial $\widehat{\theta}$-invariant functions. However bounded $\mu$-harmonic functions are constant if $\mu$ is adapted. Such a construction is as follows. For any $x \in \mathbb{Z}^{3}$, we denote

$$
\begin{aligned}
& A_{x}^{+}=\left\{\omega \in \widehat{\Omega} ; \quad \exists n>0, \quad x+S_{n}(\omega)=0\right\} \\
& A_{x}^{-}=\left\{\omega \in \widehat{\Omega} ; \quad \exists n \leq 0, \quad x+S_{n}(\omega)=0\right\} \\
& A_{x}=A_{x}^{+} \cup A_{x}^{-}, \quad A=\bigcup_{x \in \mathbb{Z}^{3}} A_{x} \times\{x\}
\end{aligned}
$$


Then $A \subset \widehat{\Omega} \times \mathbb{Z}^{3}$ is the set of paths of the random walk which pass through 0 at some time $n \in \mathbb{Z}$; clearly $A$ is $\widehat{\theta}$-invariant. On the other hand, since $S_{n}(\omega)$ is transient, if $x \neq 0$ $: 0<\widehat{\mathbb{P}}\left(A_{x}^{+}\right)<1,0<\widehat{\mathbb{P}}\left(A_{x}^{-}\right)<1$ (see [27]).

Since $S_{n}(\omega)(n>0)$ and $S_{n}(\omega)(n \leq 0)$ are independent, the complements of $A_{x}, A_{x}^{-}$ are also independent, hence $0<\left(1-\widehat{\mathbb{P}}\left(A_{x}^{+}\right)\right)\left(1-\widehat{\mathbb{P}}\left(A_{x}^{-}\right)\right)=1-\widehat{\mathbb{P}}\left(A_{x}^{+} U A_{x}^{-}\right)<1$. It follows that $A$ is a non trivial $\widehat{\theta}$-invariant set of $\widehat{\Omega} \times \mathbb{Z}^{3}$.

Definition 4 We say that the group $G$ is recurrent if there exists a probability measure $\mu$ on $G$ which is adapted and recurrent.

One can show (see below) that if $G$ is recurrent then $G$ and its closed subgroups are unimodular. Also in this case amenability of $G$ is valid and follows from the constancy of bounded harmonic functions.

\section{Examples}

a) It is well known that $\mathbb{Z}^{2}$ is recurrent while $\mathbb{Z}^{3}$ is transient (see [27]). The free group $F_{d}$ with $d$ generators $(d \geq 2)$ is transient since it is non amenable (see 8 ).

b) Let $\mathbb{G}_{2}$ be the group of motions of the euclidean plane. We identify $\mathbb{G}_{2}$ with the group of maps of the form $g z=a z+b$ where $z \in \mathbb{C}$ and $|a|=1, b \in \mathbb{C}$. Let $\alpha \notin \mathbb{Q}$ and $a, b \in \mathbb{G}_{2}$ defined by $a z=e^{2 i \pi \alpha} z+1, b z=e^{-2 i \pi \alpha} z+1, \mu=\frac{1}{2}\left(\delta_{a}+\delta_{b}\right)$. Then one can show that $\mu$ is recurrent, hence $\mathbb{G}_{2}$ is recurrent. The proof relies on the estimation of the polymer sums $S_{n}^{\prime}(\omega) 0=1+\sum_{1}^{n-1} e^{2 i \pi s_{k}(\omega)}$ where $s_{k}(\omega)=\sum_{1}^{k} \varepsilon_{k}(\omega)$ and $\varepsilon_{k}(\omega)= \pm 1$ with probability $1 / 2$; one gets $\int\left|S_{n}^{\prime}(\omega) 0\right|^{2} d \mathbb{P}(\omega) \sim C n$ with $C>0$ and this can be shown to imply recurrence (see [12]).

c) Let $G=S L(2, \mathbb{R}), \Gamma \subset G$ a cocompact discrete subgroup of $G, \Gamma^{\prime}$ a normal subgroup of $\Gamma$ such that $\Gamma / \Gamma^{\prime}=\mathbb{Z}$ or $\mathbb{Z}^{2}, E=G / \Gamma^{\prime}, \mu=\delta_{a}$ with $a=\operatorname{diag}\left(e, e^{-1}\right)$. We consider the Haar measure $m$ on $E$ and the action of $a$ on $E$. The corresponding dynamical system is the time-one geodesic flow on $E$ and can be identified with $(\widehat{\Omega} \times E, \widehat{\theta}, \widehat{\mathbb{P}} \otimes m)$. One can show (see [13], [24], [28]) that this system is ergodic. Here recurrence of the $a$-action is an easy first step based on Birkhoff ergodic theorem.

\section{Growth and recurrence}

The following quadratic growth conjecture was stated in 11]: $G$ is recurrent if and only if $G$ has polynomial growth at most 2 .

One can show using [21], that if $G$ has polynomial growth of degree at most 2 , then $G$ is recurrent (see [23]). Also for the converse one can assume that $G$ is compactly generated. In [19] it was already conjectured that finitely generated recurrent groups have non exponential growth. The general quadratic growth conjecture is based on this idea but is clearly stronger. Also its possible proof can be seen to involve necessarily a group theoretic argument. One can expect the informations of [21] to be sufficient. 
Then in the discrete case the quadratic growth conjecture has been settled in 29] using [9] : recurrent finitely generated groups are virtually $\mathbb{Z}$ or $\mathbb{Z}^{2}$. On the other hand, as shown in [1], recurrent connected Lie groups are closed subgroups of $\mathbb{G}_{2}$, up to normal compact subgroups. Then, using the analysis developed in [1], [12] and [29], one can solve the quadratic growth conjecture for Lie groups, connected or not. Also, for Lie groups over p-adic fields, the conjecture is solved in [23]. But this leaves unsolved the conjecture if $G$ is totally disconnected. As a first step, it is natural to consider the case where $G$ is a closed subgroup of $G L(V)$ with $V=\mathbb{F}^{d}$ and $\mathbb{F}$ is a non-archimedean local field. We observe that, if $\mathbb{F}$ has positive characteristic, $G$ is not a Lie group in general. In this case, due to the ultrametric triangular inequality, one can expect a much more simpler analysis and result than in [12] and 29]. This is indeed the case as shown below. Furthermore, in the general case of a recurrent compactly generated and totally disconnected group $G$, one can expect the following : $G$ has an open compact normal subgroup $K$ such that $G / K$ is recurrent and finitely generated. Then, the corresponding result would follow from [29].

\section{$5 \quad$ Linear groups over local fields}

One has the following.

$\underline{\text { Theorem } 1}$ Assume $G$ is a closed subgroup of a finite product of $\prod_{i \in I} G L\left(d_{i}, \mathbb{F}_{i}\right)$ where any $\mathbb{F}_{i}$ is a local field. Then $G$ is recurrent if and only if $G$ has at most quadratic growth.

One will sketch the main ideas of the proof of necessity of quadratic growth in the case $I=\{1\}$ and $\mathbb{F}_{1}=\mathbb{F}$ a non-archimedean local field. Also one will assume $G$ compactly generated recurrent and show the existence of a compact normal subgroup $K$ such that $G / K$ is virtually $\mathbb{Z}$ or $\mathbb{Z}^{2}$. One will need to consider the contraction subgroup $C_{g}$ of $g \in G$ i.e

$$
C_{g}=\left\{h \in G ; \lim _{n \rightarrow \infty} g^{n} h g^{-n}=e\right\}
$$

If $G$ is a closed linear group it is easy to show that $C_{g}$ is also closed. Furthermore if $C_{g} \neq\{e\}$ is closed, the closed subgroup of $G$ generated by $g$ and $C_{g}$ is not unimodular. Then the two main steps are given by the following propositions.

Proposition 2 Assume $G$ is locally compact and recurrent. Then every closed subgroup of $G$ is unimodular.

It follows from the above remark that, if furthermore $G \subset G L(V)$ is closed, then $C_{g}=C_{g^{-1}}=\{e\}$

Proposition 3 Assume $G$ is a compactly generated closed subgroup of $G L(d, \mathbb{F})$ where $\mathbb{F}$ is a non archimedean local field. Then, if $C_{g}=\{e\}$ for any $g \in G, G$ has a compact open normal subgroup.

In the proof of this result an important role is played by the following lemmas. 
Lemma 1 (See [18])

Assume $G$ is totally disconnected and $g \in G$ satisfies $C_{g}=C_{g^{-1}}=\{e\}$. Then there exists a compact open subgroup $K_{g}$ such that $g K_{g} g^{-1}=K_{g}$.

This lemma is a consequence of the study of the so called tidy subgroups [2].

Lemma 2 (See [3], 17])

Assume $G \subset G L(d, \mathbb{F})$ is compactly generated, $\mathbb{F}$ is non-archimedean, and any $g \in G$ generates a bounded subgroup. Then $G$ is bounded.

One may observe that, on the real field, even if $G$ is finitely generated, the corresponding statement is false, which gives a negative answer to a question of S. Ulam (see [3]). But, on ultrametric fields, S. Ulam's question has a positive answer. On the other hand, if $G$ is assumed to be closed, the conclusion of lemma 2 is valid for any local field (see [3], [17]).

Let us give the proof of Proposition 2. Let $\mu$ be adapted and recurrent on $G$. Then, if we denote by $P$ the Markov operator on $E=G / H$ associated to $\mu$, we have for any

$u \in C_{c}^{+}(E), \quad u \neq 0: \sum_{0}^{\infty} P^{k} u=+\infty$ on $E$. Hence, using [20], one sees that there exists a $P$-invariant Radon measure $\lambda$ on $E$. For any $\varphi \in C_{c}^{+}(E)$, we consider the non negative function $f$ on $G$ given by $f(g)=g \lambda(\varphi)$. Clearly $f(g)=\int f(g h) d \mu(h)$, i.e $f$ is $\mu$ harmonic on $G$. Since $\mu$ is recurrent and adapted, Proposition 1 implies that $f$ is constant i.e $g \lambda(\varphi)=\lambda(\varphi)$. Since $g$ and $\varphi$ are arbitrary $\lambda$ is $G$-invariant. As is well known from general group theory this implies $\Delta_{G}(h)=\Delta_{H}(h)$ for any $h \in H$, where $\Delta_{G}\left(\operatorname{resp} \Delta_{H}\right)$ is the modular function of $G$ (resp $H$ ). If we take for $H$ the closed subgroup generated by $g \in G$, we get $\Delta_{G}(g)=\Delta_{H}(g)=1$; hence $G$ is unimodular. From above it follows $\Delta_{H}(h)=1$ for any $h \in H$, hence $H$ is unimodular.

\section{Homogeneous spaces}

Various examples of recurrent and ergodic behaviours take place (see [5], [17], [12]). Here we only develop one example and formulate a general question.

1) A framework

Let $E$ be a locally compact $G$-space, $P$ the Markov operator on $C_{b}(E)$ defined by :

$$
P \varphi(x)=\int \varphi(g x) d \mu(g),
$$

we consider a $P$-invariant Radon measure $\lambda$ on $E$, i.e $P \lambda=\lambda$. Then we can consider the extended shifts on $\Omega \times E$ and $\widehat{\Omega} \times E$ defined by $\widetilde{\theta}(\omega, x)=\left(\theta \omega, X_{1}(\omega) x\right)$ and $\widehat{\theta}(\omega, x)=$ $\left(\theta \omega, X_{1}(\omega) x\right)$, we endow $\Omega \times E$ and $\widehat{\Omega} \times E$ with their natural structure of Polish spaces so that $\widetilde{\theta}$ is continuous and $\widehat{\theta}$ is a homeomorphim we write $\Omega^{-}=G^{-\mathbb{N} \cup\{0\}}$. Then the Markov measure $\widetilde{\lambda}=\mathbb{P} \otimes \lambda$ on $\Omega \times E$ is $\widetilde{\theta}$-invariant. One can show that there exists on $\widehat{\Omega} \times E$ a unique Radon measure $\widehat{\lambda}$ which is $\widehat{\theta}$-invariant and has projection $\widetilde{\lambda}$ on $\Omega \times E$. 
In the general case of a dynamical system with $\sigma$-finite invariant measure, the construction goes back to V.A Rokhlin ( [25]). In our special situation, $\widehat{\lambda}$ can be defined as follows. Let $P^{*}$ be the adjoint operator of $P$ in $\mathbb{L}^{2}(E, \lambda)$. Since $P \lambda=\lambda, P^{*}$ is also a Markov operator and we denote by $\stackrel{\vee}{\mathbb{P}}_{\lambda}$ the corresponding Markov measure on $\Omega^{-} \times E$. If we denote by $\theta^{-}$ the shift on $\Omega^{-}$, we can define an extended shift on $\Omega^{-} \times E$ by $\widetilde{\theta}^{-}\left(\omega_{-}, x\right)=\left(\theta^{-} \omega_{-}, g_{0}^{-1} x\right)$. Then $\stackrel{\vee}{\mathbb{P}} \lambda$ is $\widetilde{\theta}^{-}$-invariant and has projection $\lambda$ on $E$, hence we can disintegrate $\stackrel{\vee}{\mathbb{P}_{\lambda}}$ as $\stackrel{\vee}{\mathbb{P}}_{\lambda}=\int \stackrel{\vee}{\mathbb{P}}_{y} \otimes \delta_{y} d \lambda(y)$, along the fibers $\Omega^{-} \times\{y\}$. Then one can verify that the measure $\widehat{\lambda}=\int \stackrel{\vee}{\mathbb{P}}_{y} \otimes \mathbb{P} \otimes \delta_{y} d \lambda(y)$ is $\widehat{\theta}$-invariant.

\section{Definitions 5}

Let $(E, P, \lambda)$ be as above. Then one says that $(E, P, \lambda)$ has property $R$ if for any relatively compact open set $U \subset E$, and $\mathbb{P} \otimes \lambda$-a.e $(\omega, x) \in \Omega \times U$, we have $S_{n}(\omega) x \in U$ infinitely often.

If $E=G, \lambda=\lambda_{G}$ and $P$ is as above then property $R$ for $(E, P, \lambda)$ is equivalent to recurrence of $\mu$ on $G$. Then one has the

\section{Proposition 4}

Assume $E$ is not the $G$-space $\mathbb{Z}$ with $\mu=\delta_{g}$ acting by translation on $\mathbb{Z}$. Then one has the equivalence

a) Property $R$ is valid for $(E, P, \lambda)$ and the equation $P f=f, f \in \mathbb{L}^{\infty}(\lambda)$ implies $f$ is constant.

b) Property $R$ is valid for $(E, P, \lambda)$ and $\lambda$ is extremal in the cone of $P$-invariant Radon measures.

c) The homeomorphism $\widehat{\theta}$ is ergodic on $\widehat{\Omega} \times E$ with respect to $\widehat{\lambda}$.

2) An example

Assume $G$ is semisimple of real rank $1, \Gamma$ is a discrete cocompact subgroup and $\Gamma^{\prime}$ is a normal subgroup of $\Gamma$ which satisfies $\Gamma / \Gamma^{\prime}=\mathbb{Z}$. We endow $E=G / \Gamma^{\prime}$ with the Haar measure $\lambda=m$ and observe that $G / \Gamma^{\prime}$ is an abelian cover of the compact homogeneous space $G / \Gamma$. We consider the homeomorphism $\widehat{\theta}$ of $\widehat{\Omega} \times E$ and the measure $\widehat{\mathbb{P}} \otimes m=\widehat{\lambda}$

\section{Proposition 5}

With the above notations we assume $\mu$ is symmetric with compact support and $G_{\mu}$ is non amenable. Then $\widehat{\theta}$ is ergodic with respect to $\widehat{\mathbb{P}} \otimes m$.

For the proof we use the equivalence of a) and c) in the above proposition. Property $R$ follows easily from the symmetry of $\mu$ and the $G$-invariance of $m$, using ergodicity of the random walk on $G / \Gamma$.

For the study of the equation $P f=f, f \in \mathbb{L}^{\infty}(E)$, one uses induced unitary representations, as follows.

One observes that $\Gamma / \Gamma^{\prime}=\mathbb{Z}$ acts on $G / \Gamma^{\prime}$ and this action commutes with the $G$-action. Taking a Borel fundamental domain $\Delta \subset E$ of this action one can identify $G / \Gamma^{\prime}$ with $\Delta \times \mathbb{Z}$. One denotes by $x \rightarrow g . x$ the transformation of $G / \Gamma$ defined by $g \in G$. Then for $x \in \Delta \subset E$ 
one can write : $g x=(g \cdot x) z(g x)$ with $z(g x) \in \mathbb{Z}, g \cdot x \in \Delta$. For any character $\chi$ of $\Gamma / \Gamma^{\prime}=\mathbb{Z}$ one can define a unitary representationn $\rho_{\chi}$ of $G$ in $\mathbb{L}^{2}(G / \Gamma)$ by

$$
\rho_{\chi}(g) f(x)=f\left(g^{-1} \cdot x\right) \chi\left(z\left(g^{-1} x\right)\right)
$$

If $\chi(\Gamma)=1$, i.e $\chi=0$ we get the natural representation $\rho_{0}$ of $G$ in $\mathbb{L}^{2}(G / \Gamma)$ and we know that $\rho_{0}$ restricted to $\mathbb{L}_{0}^{2}(G / \Gamma)$ do not contain weakly the identity representation. Since the one dimensional representation of $\Gamma$ defined by $\chi$ do not contain weakly identity, the same is valid for the induced representation $\rho_{\chi}$ from $\Gamma$ to $G$ (see [22] Prop 1-11 p. 112). Then one can use the non-amenability of $G_{\mu}$ and a result of [25] (see also [4], [16]) to conclude that if $\chi \neq 0$ the operator $\rho_{\chi}(\mu)$ defined by $\rho_{\chi}(\mu) f(x)=\int f(g x) \chi[z(g x)] d \mu(g)$ satisfies $\left\|\rho_{\chi}(\mu)\right\|<1$.

Then the same analysis as in 15] can be performed with the natural Fourier decomposition of $\mathbb{L}^{2}(E)$ : we get that the condition $P f=f, f \in \mathbb{L}^{\infty}(E)$ implies $f=$ cte.

\section{3) A question}

Let $G$ be a connected Lie group or an algebraic group defined over a local field $\mathbb{F}, H$ a closed subgroup, $\lambda$ a $P$-invariant Radon measure on $E=G / H, x \in \operatorname{supp} \lambda$. Assume that $(E, P, \lambda)$ satisfies property $R$. Then, is it true that, for any $n \in N: \lambda\left(W^{n} x\right) \leq C_{W} n^{2}$, where $W$ is a compact neighbourhood of e and $C_{W}>0$ ?

Is it possible to describe geometrically the systems $(E, P, \lambda)$ if $\lambda$ is finite ? Is it true that the basic building blocks for such an $E$ are either boundaries of $(G, \mu)$ or spaces of the form $G / \Gamma$ where $\Gamma$ is a lattice in $G$ ?

\section{References}

[1] P. Baldi. Caractérisation des groupes de Lie connexes récurrents, Ann. Inst. H. Poincaré Sect. B (N.S.) 17 (1981), 281-308.

[2] U. Baumgartner and Willis. Contraction groups and scales of automorphisms of totally disconnected locally compact groups, Israel J. Math. 142 (2004), 221-248.

[3] J-P Conze and Y. Guivarc'h. Remarques sur la distalité dans les espaces vectoriels, C.R. Acad. Sci. Paris Sr. A 278 (1974), 1083-1086.

[4] Y. Derriennic and Y. Guivarc'h. Théorème de renouvellement pour les groupes non moyennables, C.R.A.S. Paris 277 (1973) A 613-A 615.

[5] A. Eskin and G.A. Margulis. Recurrence properties of random walks on finite volume homogeneous manifolds, Random walks and geometry, 431-444, Walter de Gruyter, Berlin 2004.

[6] S.R. Foguel. The ergodic theory of Markov processes. Van Nostrand Mathematical Studies, No. 21 Van Nostrand Reinhold Co., New York-Toronto, Ont.-London 1969. 
[7] A. Furman. Random walks on groups and random transformations. Handbook of dynamical systems, Vol. 1A, 931-1014, North-Holland, Amsterdam, 2002.

[8] H. Furstenberg. Random walks and discrete sugroups of Lie groups. Advances in Probability and Related topics (Ed. P. Ney) vol. 1, p. 1-63, M. Dekker (1971).

[9] M. Gromov. Groups of polynomial growth and expanding maps, I.H.E.S. Publ. Math. No. (1981), 53-73.

[10] Y. Guivarc'h. Croissance polynomiale et périodes des fonctions harmoniques, Bull. Soc. Math. France, 101 (1973), 333-379.

[11] Y. Guivarc'h and M. Keane. Marches aléatoires transitoires et structure des groupes de Lie. Symposia Mathematica, Vol. XXI (Convegno sulle Misure su Gruppi e su Spazi Vettoriali, Convegno sui Gruppi e Anelli Ordinati, INDAM, Rome, 1975), 197-217. Academic Press, London 1977.

[12] Y. Guivarc'h , M. Keane and B. Roynette. Marches aléatoires sur les groupes de Lie, Lecture Notes in Mathematics 624, Springer-Verlag, Berlin-New York, 1977.

[13] Y. Guivarc'h. Propriétés ergodiques, en mesure infinie, de certains systèmes dynamiques fibrés, Ergodic Theory Dynam. Systems 9 (1989), 433-453.

[14] Y. Guivarc'h. Marches aléatoires sur les groupes, Development of Mathematics 19502000, 577-608, Birkhauser, Basel, 2000.

[15] Y. Guivarc'h and A.N. Starkov. Orbits of linear group actions, random walks on homogeneous spaces and toral automorphisms. Ergodic Theory Dynam. Systems 24 (2004), 767-802.

[16] Y. Guivarc'h. Limit Theorems for Random walks and Products of Random Matrices. In Probability Measures on Groups Eds : S.G. Dani and P. Graczyk, p 256-33, 2006 T.I.F.R. Narosa Publ. House, New Delhi, India.

[17] Y. Guivarc'h and C.R.E. Raja. Recurrence and Ergodicity of random walks on locally compact groups and on homogeneous spaces. Preprint 2009.

[18] W. Jaworksi and C.R.E. Raja. The Choquet-Deny theorem and distal properties of totally disconnected locally compact groups of polynomial growth, New York J. math 13 (2007), 159-174.

[19] A. Kesten. The Martin boundary of recurrent random walks on countable groups 1967 Proc. Fifth Berkeley Sympos. Math. Statist. and Probability (Berkeley, Calif., 1965/66) Vol. II ; Contributions to Probability Theory, Part 2, pp. 51-74. Univ California Press, Berkeley, Calif. 
[20] M. Lin. Conservative Markov processes on a topological space, Israel J. Math. 8 (1970), 165-186.

[21] V. Losert. On the structure of groups with polynomial growth. Mzth. Z. 195 (1987), 109-117.

[22] G. Margulis. Discrete subgroups of semisimple Lie groups, Ergebnisse der Mathematik und ihrer Grenzgebiete (3) 1. Springer-Verlag, Berlin, 1991.

[23] C.R.E. Raja. On growth, recurrence and the Choquet-Deny Theorem for $p$-adic Lie groups, Math. Z. 251 (2005), 827-847.

[24] M. Rees. Checking ergodicity of some geodesic flows with infinite Gibbs measure, Ergodic Theory Dynam. Systems 1 (1981), 107-133.

[25] V.A Rokhlin Selected topics from the metric theory of dynamical systems Uspehi Mat. Nauk. 4 (1949), 57-125 A.M.S. Transl. Ser. 249(1966).

[26] Y. Shalom. Explicit Kazhdan constants for representations of semisimple and arithmetic groups, Annales de l'institut Fourier, 50 (1979), 171-202.

[27] F. Spitzer. Principles of Random walk, Van Nostrand, 1964.

[28] D. Sullivan. The density at infinity of a discrete group of hyperbolic motions, I.H.E.S. Publ. Math. No 50 (1979), 171-202.

[29] Th. Varopoulos, L. Saloff-Coste and T. Coulhon. Analysis and geometry on groups, Cambridge Tracts in Mathematics, 100. Cambridge University Press, Cambridge, 1992.

[30] W. Woess. Random walks on infinite graphs and groups, Cambridge Tracts in Mathematics, 138. Cambridge University Press, Cambridge, 2000.

$\begin{array}{ll}\text { Yves Guivarc'h } & \text { C.R.E. Raja } \\ \text { IRMAR } & \text { Stat. Math. Unit. } \\ \text { Université de Rennes I } & \text { Indian Statistical Institute } \\ \text { Campus de Beaulieu } & \text { 8th Mile Mysore Road } \\ \text { 35042 Rennes Cedex, France } & \text { Bangalore 560059 } \\ \text { yves.guivarch@univ-rennes1.fr } & \text { creraja@isibang.ac.in }\end{array}$

